\title{
Scenario in nanotechnology research
}

\begin{abstract}
There is a range of properties that are appering due to the fact that some structures having nanometric dimensions, resulting in a high surface area, a greater degree of dispersion and functionalities that depend on the size of the structure. There is a big concern about reducing the environmental impact of human activities in recent decades that leads to a greater demand for processes and materials with high environmental performance. High environmental performance means lower energy requirements, less consumption of non-renewable inputs and less waste. Increasingly stringent environmental legislation, greater environmental awareness on the part of the population, and government agents involved in environmental protection require the development of processes and materials with high environmental performance. In this way, a great opportunity presents itself to Brazil as new technological processes and advanced materials are developed, promoting the exploration of mineral and biological wealth in an environmentally sustainable way.
\end{abstract}

Volume 3 Issue $6-2018$

\author{
Nulliany da Silva,' Isabel Dinóla, ${ }^{2}$ Minos A \\ Neto' \\ 'Department of Physics, Federal University of Amazonas, Brazil \\ ${ }^{2}$ Center for Analysis Foundation, Research and Technological \\ Innovation, Brazil
}

Correspondence: Minos Martins Adão Neto, Department of Physics, Federal University of Amazonas, 3000, Japiim, 69077-000, Manaus-AM, Brazil, Email minas@pq.cnpq.br

Received: January II, 2018 | Published: November 23, 2018

\section{The Scenario}

What differentiates the nano materials from the others is that they allow enhancing physical and chemical properties in extremely low concentrations, giving new characteristics to a given product, which in the past was not possible. This range of properties is basically due to the fact that these structures have nanometric dimensions, resulting in a high surface area, a greater degree of dispersion and functionalities that depend on the size of the structure.

The nanotechnology research that is being developed in the country indicates that business opportunities arise at first in the cosmetics, chemicals from the chemical industry (catalysts, paints, coatings) and petrochemicals, plastics, rubbers and metal alloys.

The generation of inputs for cosmetics, for example, is an opportunity for the enrichment of agricultural chains with new products. Nowadays, companies in the agro sector have the challenge of meeting the increasingly demanding consumer, who demands products with low environmental impact.

This growing concern about reducing the environmental impact of human activities in recent decades leads to a greater demand for processes and materials with high environmental performance. High environmental performance means lower energy requirements, less consumption of non-renewable inputs and less waste. In addition, the presence of environmental liabilities in degraded areas resulting from industrial activities needs to be properly managed to reduce their effects on the environment. Increasingly stringent environmental legislation, greater environmental awareness on the part of the population, and government agents involved in environmental protection require the development of processes and materials with high environmental performance. In this way, a great opportunity presents itself to Brazil as new technological processes and advanced materials are developed, promoting the exploration of mineral and biological wealth in an environmentally sustainable way. ${ }^{1}$

Any anthropogenic activities generate several wastes that contaminate rivers, lakes and aquifers. These contaminations can cause various harmful effects on the environment and human health. Studies have identified the importance of Brazil in providing solutions to such problems, provided they make the necessary investments in the development of advanced ceramic, polymeric and hybrid adsorbent materials. In addition, the development of these materials contributes to the sustainability of several important industrial segments. Functional materials have features designed to serve for specific purposes in a controlled manner. Its physical and chemical properties are sensitive to changes in the environment such as temperature, pressure, electric or magnetic field, radiation wavelength, $\mathrm{pH}$, presence of gases and other substances. Due to their characteristic properties and functions, the functional materials behave intelligently in response to stimulus of the medium to fulfill specific functions.

With nanotechnology, it became possible to manufacture sensors, which are devices that respond to a specific and measurable, smaller, more selective and more sensitive physical/chemical stimulus for the detection and monitoring of organic and inorganic pollutants in the environment. Most of the sensors are obtained from polymeric, ceramic or semiconductor materials. Functionalized conducting polymers find increasing commercial application in the manufacture of instruments for applications as diverse as biomolecules detection, chemical compounds (chemical sensors) and energy storage. Advances in sensors for the detection of pollutants imply directly in better control of industrial processes and in earliest and accurate detection of the existence of problems of contamination.

In general, the improved catalyst/adsorbent performance is associated with smaller particles, which give the material high specific surface areas. Nanoscale adsorbents and catalysts are designed to have a high capacity to immobilize contaminating species on their surface. However, the smaller the adsorbent particles and/or the catalyst, the more difficult it is to separate the aqueous solution. Thus, a solution is the use of magnetic nanoparticles, so that magnetic separation is used, which is an efficient technique in the separation of these particles and is used for several applications in areas such as biochemistry, medicine, analytical chemistry, mining and environmental engineering.

In particular, the super paramagnetic nanoparticles of iron oxide have received considerable attention in the last few years due to the possibility of controlling them, allowing, for example, the delivery of drugs within the human body in a certain affected area.

The magnetic particles can combine with functional groups or inorganic compounds, forming the magnetic composites and the 
challenge of separating adsorbents/catalysts with high specific surface areas of solutions can be solved with the use of magnetic composites. Magnetic composites can be conveniently recovered by magnetic separation, avoiding the filtration steps, which represent a barrier for the application of high performance materials in environmental recovery processes and in the treatment of large volumes of aqueous solutions. A series of magnetic composites, which can be separated by magnetic separation processes, is used to remove a wide variety of contaminants from water. Carbon magnetic composites, ${ }^{2,3}$ magnetic adsorbents of chitosan and alginate, synthetic magnetic polymers, clays and magnetic metal oxides exhibiting a large specific surface area or selective functional groups have been developed. These magnetic composites are obtained by mixing materials, at least one of which is magnetic. These materials have a great potential to remove organic and inorganic pollutants in absorption processes due to their high absorptive capacities and convenient magnetic separation. Magnetic manganese oxide composites can be viewed as potential absorbents/catalysts, since they combine properties and oxidative reactions of manganese oxides with the ease of recovery of magnetic materials. Figure 1 briefly shows the composition of the composite that involves the precipitation of manganese oxide from the reaction between manganese (II) chloride and air oxygen in the presence of magnetite particles. The magnetic composites efficiently oxidized As (III) (the most toxic and mobile arsenic species) to As (V) (less toxic and mobile species), facilitating the removal of the most toxic species from the solution by applying a magnetic field.

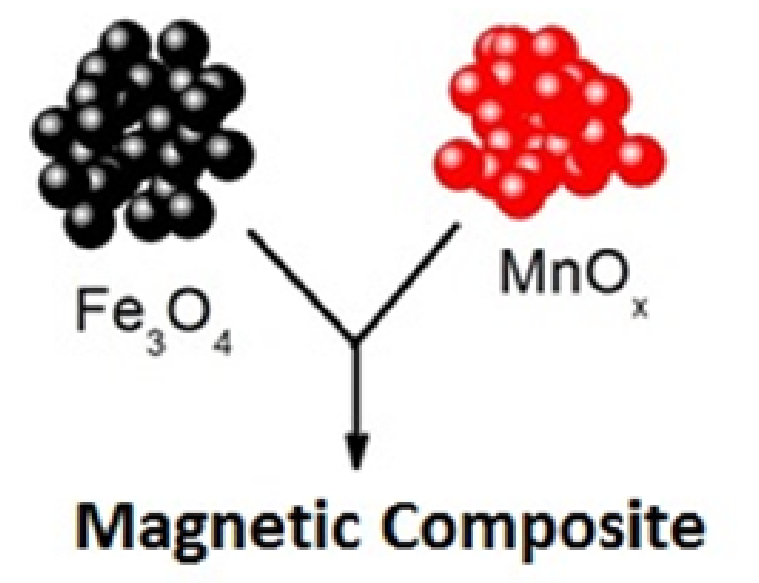

Figure I Magnetic composite.
The constant challenge of making industrial processes less impactful to the environment encourages the development of advanced materials. The knowledge of the physical and chemical properties of the species, the mechanism of heterogeneous chemical reactions, the electronic and geometric structure of the materials and the thermodynamics of the solid formation processes is fundamental for the development of nanotechnology. Advanced materials are developed with increasingly specific objectives such as gas detection, gas storage, catalysis, metal retention, mechanical strength, molecular sieves, electronic devices, light converters, among other applications. In the case of the mineral extraction industry and the environment, studies point to an economic and strategic opportunity for the development of innovative materials (ceramic, polymer, hybrids and others) with functions of separation, immobilization and sequestration of polluting substances. Brazil has a strategic advantage for the abundance of raw materials and inputs for the production of these materials on a large scale.

\section{Acknowledgments}

None.

\section{Conflicts of interest}

Author declares there is no conflicts of interest.

\section{References}

1. Ministry of Environment. Water, an increasingly threatened resource. 2015.

2. Rodrigues APH. Study of the adsorption of cadmium ion from the aqueous medium using magnetic composite $\mathrm{Mn}_{3} \mathrm{O}_{4} / \mathrm{Fe}_{3} \mathrm{O}_{4}$. Belo Horizonte - MG; 2014

3. Silva GC. Development of nano-sized Mn3O4 magnetic composites: application in wastewater treatment. Belo Horizonte - MG; 2012. 